\title{
Adomian Decomposition Method Used to Solve a SIR Epidemic Model of Dengue Fever
}

\author{
Yulius Keremata Lede ${ }^{1}$, Sudi Mungkasi ${ }^{2}$ \\ \{yuliusllede@gmail.com¹, sudi@usd.ac.id² $\}$ \\ Department of Mathematics Education, Faculty of Teacher Training and Education, Universitas \\ Sanata Dharma, Yogyakarta, Indonesia ${ }^{1}$ \\ Department of Mathematics, Faculty of Science and Technology, Universitas Sanata Dharma, \\ Yogyakarta, Indonesia ${ }^{2}$
}

\begin{abstract}
In this paper, we solve a SIR (Susceptible-Infectious-Recovered) epidemic model of dengue fever. The SIR model is difficult to solve exact-analyticaly. Therefore, we use an approximate method. The method that we use is the Adomian decomposition method. The method produces approximate solutions as series. With only three terms in the series, we observe that the approximate solutions are observed. This means that the Adomian decomposition method can be used in solving the SIR model.
\end{abstract}

Keywords: Adomian decomposition method, dengue fever, SIR model

\section{Introduction}

The spread of dengue fever can be considered as a study in biology. However, the spread of dengue fever can be studied through mathematics using mathematical models. One available model is the SIR (Susceptible-Infectious-Recovered) epidemic model.

A number of researchers have studied the SIR epidemic model of dengue fever. Side and Noorani [1] simulated the spread of dengue fever disease for South Sulawesi, Indonesia and Selangor, Malaysia regions. Rangkuti et al. [2] proposed homotopy perturbation method and variational iteration method for solving the SIR model.

To complete the studies of those researchers [1]-[2], we shall use Adomian decomposition method. Putranto and Mungkasi [3] used Adomian decomposition method for solving a population dynamics model involving two species. The SIR model that we shall consider in this paper involves three groups of populations. In this paper, we implement Adomian decomposition method for solving the SIR model of dengue. We use the Adomian decomposition method, because it is a reliable method, as has been studied by Wazwaz [4].

This paper is structured as follows: Section 2 will discuss the mathematical model, Section 3 will discuss the Adomian decomposition method used to solve the mathematical model, Section 4 will provide mathematical results of computations, and Section 5 will give conclusions.

\section{Mathematical model}

Side and Noorani [1] as well as Rangkuti et al. [2] defined the dengue fever SIR model in the following system of equations: 


$$
\begin{gathered}
\frac{d x}{d t}=\mu_{h}(1-x(t))-\alpha x(t) z(t) \\
\frac{d y}{d t}=\alpha x(t) z(t)-\beta y(t) \\
\frac{d z}{d t}=\gamma(1-z(t)) y(t)-\delta_{1} z(t)
\end{gathered}
$$

where $x=\frac{s_{h}}{N_{h}}, \quad y=\frac{I_{h}}{N_{h}}, \quad z=\frac{I_{v}}{N_{v}}=\frac{I_{v}}{A / \mu_{h}}, \quad \alpha=\frac{b \beta_{h} A}{\mu_{v} N_{h}}, \quad \beta=\gamma_{h}+\mu_{h}$ and $\gamma=b \beta_{v}, \delta_{1}=\mu_{v}$. Here $\gamma_{h}, b \beta_{v}, b \beta_{h}, \mu_{h}$ and $\mu_{v}$ are parameters, $t$ is the time variable, $A$ is the number of mosquito population births, $N_{h}$ is the human population, $s_{h}$ is the number of people who are potentially infected with the dengue virus, $I_{h}$ is the number of people who are infected with dengue, $R_{h}$ is the number of people who have recovered after infection. The number of mosquito population as the virus vectors $\left(N_{v}\right)$ is divided into two groups: mosquitoes that are potentially infected with dengue virus (susceptible; $s_{v}$ ) and mosquitoes infected with dengue virus $\left(I_{v}\right) . b \beta_{h}$ is a sufficient level of correlation between vector populations and human populations.

\section{Adomian decomposition method}

Let us consider the dengue SIR model (1)-(3). We rewrite the system of equations (1)-(3) to:

$$
\begin{gathered}
\frac{d x}{d t}=\mu_{h}-\mu_{h} x-\alpha x z \\
\frac{d y}{d t}=\alpha x z-\beta y \\
\frac{d z}{d t}=\gamma y-\gamma y z-\delta_{1} z
\end{gathered}
$$

Furthermore, we assume to have the initial condition $x(0)=x_{0} \equiv c_{1}, \quad y(0)=y_{0} \equiv c_{2}$ and $z(0)=z_{0} \equiv c_{3}$.

Following Wazwaz [4], we use the operator notation $L=\frac{d}{d t}$ then the system of equations (4)-(6) becomes:

$$
\begin{gathered}
L_{x}=\mu_{h}-\mu_{h} x-\alpha x z \\
L_{y}=\alpha x z-\beta y \\
L_{z}=\gamma y-\gamma y z-\delta_{1} z
\end{gathered}
$$

If the inverse operator $L^{-1}=\int_{0}^{t}() d$.$t is applied to the both sides of each equations, the$ system of nonlinear equations becomes:

$$
\begin{gathered}
L^{-1} L_{x}=L^{-1} \mu_{h}-L^{-1} \mu_{h} x-L^{-1} \alpha x z \\
L^{-1} L_{y}=L^{-1} \alpha x z-L^{-1} \beta y \\
L^{-1} L_{z}=L^{-1} \gamma y-L^{-1} \gamma y z-L^{-1} \delta_{1} z
\end{gathered}
$$


The Adomian decomposition method decomposes $x, y$ and $z$ into an infinite number of components, so that they become:

$$
x(t)=\sum_{0}^{\infty} x_{n}, \quad y(t)=\sum_{0}^{\infty} y_{n}, \quad z(t)=\sum_{0}^{\infty} z_{n},
$$

and for non-linear components of the system equation (10)-(12) namely $x z$ and $y z$ to be:

$$
x z=\sum_{0}^{\infty} A_{n}, y z=\sum_{0}^{\infty} B_{n} .
$$

The sum of nonlinear components can be described as follows:

where $k=0,1,2, \ldots, n$.

$$
A_{n}=\sum_{0}^{\infty} x_{k} z_{n-k}, B_{n}=\sum_{0}^{\infty} y_{k} z_{n-k}
$$

The Adomian polynomials $A_{n}$ and $B_{n}$ are obtained as follows:

$$
\begin{aligned}
& A_{0}=x_{0} z_{0} \\
& A_{1}=x_{0} z_{1}+x_{1} z_{0} \\
& A_{2}=x_{0} z_{2}+x_{1} z_{1}+x_{2} z_{0} \\
& A_{3}=x_{0} z_{3}+x_{1} z_{2}+x_{2} z_{1}+x_{3} z_{0} \\
& \cdots \\
& B_{0}=y_{0} z_{0} \\
& B_{1}=y_{0} z_{1}+y_{1} z_{0} \\
& B_{2}=y_{0} z_{2}+y_{1} z_{1}+y_{2} z_{0} \\
& B_{3}=y_{0} z_{3}+y_{1} z_{2}+y_{2} z_{1}+y_{3} z_{0}
\end{aligned}
$$

Substitution these results into the system of equations (10)-(12) leads to:

$$
\begin{gathered}
x(t)-x(0)=L^{-1} \mu_{h}-L^{-1} \mu_{h} \sum_{0}^{\infty} x_{n}-L^{-1} \alpha \sum_{0}^{\infty} A_{n} \\
y(t)-y(0)=L^{-1} \alpha \sum_{0}^{\infty} A_{n}-L^{-1} \beta \sum_{0}^{\infty} y_{n} \\
z(t)-z(0)=L^{-1} \gamma \sum_{0}^{\infty} y_{n}-L^{-1} \gamma \sum_{0}^{\infty} B_{n}-L^{-1} \delta_{1} \sum_{0}^{\infty} z
\end{gathered}
$$

or

$$
\begin{gathered}
\sum_{0}^{\infty} x_{n}-x(0)=L^{-1} \mu_{h}-L^{-1} \mu_{h} \sum_{0}^{\infty} x_{n}-L^{-1} \alpha \sum_{0}^{\infty} A_{n} \\
\sum_{0}^{\infty} y_{n}-y(0)=L^{-1} \alpha \sum_{0}^{\infty} A_{n}-L^{-1} \beta \sum_{0}^{\infty} y_{n} \\
\sum_{0}^{\infty} z-z(0)=L^{-1} \gamma \sum_{0}^{\infty} y_{n}-L^{-1} \gamma \sum_{0}^{\infty} B_{n}-L^{-1} \delta_{1} \sum_{0}^{\infty} z
\end{gathered}
$$

or 


$$
\begin{gathered}
\sum_{0}^{\infty} x_{n}=x(0)+L^{-1} \mu_{h}-L^{-1} \mu_{h} \sum_{0}^{\infty} x_{n}-L^{-1} \alpha \sum_{0}^{\infty} A_{n} \\
\sum_{0}^{\infty} y_{n}=y(0)+L^{-1} \alpha \sum_{0}^{\infty} A_{n}-L^{-1} \beta \sum_{0}^{\infty} y_{n} \\
\sum_{0}^{\infty} z=z(0)+L^{-1} \gamma \sum_{0}^{\infty} y_{n}-L^{-1} \gamma \sum_{0}^{\infty} B_{n}-L^{-1} \delta_{1} \sum_{0}^{\infty} z
\end{gathered}
$$

The initial values are $x(0)=x_{0}, y(0)=y_{0}, z(0)=z_{0}$. We obtain, as a result:

$$
\begin{gathered}
x_{0}+x_{1}+x_{2}+\cdots=x(0)+L^{-1} \mu_{h}-L^{-1} \mu_{h}\left(x_{0}+x_{1}+x_{2}+\cdots\right) \\
-L^{-1} \alpha\left(A_{0}+A_{1}+A_{2}+\cdots\right) \\
y_{0}+y_{1}+y_{2}+\cdots=y(0)+L^{-1} \alpha\left(A_{0}+A_{1}+A_{2}+\cdots\right) \\
-L^{-1} \beta\left(y_{0}+y_{1}+y_{2}+\cdots\right. \\
\\
z_{0}+z_{1}+z_{2}+\cdots=z(0)+L^{-1} \gamma\left(y_{0}+y_{1}+y_{2}+\cdots\right) \\
-L^{-1} \gamma\left(B_{0}+B_{1}+B_{2}+\cdots\right) \\
-L^{-1} \delta_{1}\left(z_{0}+z_{1}+z_{2}+\cdots\right)
\end{gathered}
$$

By observing the two sides of equation (27), it is obtained:

$$
\begin{gathered}
x_{0}=x(0)+L^{-1} \mu_{h} \\
x_{1}=-L^{-1} \mu_{h} x_{0}-L^{-1} \alpha A_{0} \\
x_{2}=-L^{-1} a \mu_{h}-L^{-1} \alpha A_{1}
\end{gathered}
$$

By observing the two sides of equation (28), it is obtained:

$$
\begin{gathered}
y_{0}=y(0) \\
y_{1}=L^{-1} \alpha A_{0}-L^{-1} \beta y_{0} \\
y_{2}=L^{-1} \alpha A_{1}-L^{-1} \beta y_{1}
\end{gathered}
$$

Further, equation (29) gives results:

$$
\begin{gathered}
z_{0}=z(0) \\
z_{1}=L^{-1} \gamma y_{0}-L^{-1} \gamma B_{0}-L^{-1} \delta_{1} z_{0} \\
z_{2}=L^{-1} \gamma y_{1}-L^{-1} \gamma B_{1}-L^{-1} \delta_{1} z_{1}
\end{gathered}
$$

In general, equations (30)-(32) have the forms:

$$
\begin{gathered}
x_{0}=x(0)+L^{-1} \mu_{h}, \quad x_{n+1}=-L^{-1} \mu_{h} x_{n}-L^{-1} \alpha A_{n} \\
y_{0}=y(0), \quad y_{n+1}=L^{-1} \alpha A_{n}-L^{-1} \beta y_{n} \\
z_{0}=z(0), \quad z_{n+1}=L^{-1} \gamma y_{n}-L^{-1} \gamma B_{n}-L^{-1} \delta_{1} z_{n}
\end{gathered}
$$

In general, the solution of the equation system is the sum of all iterations. However, we can determine the number of iterations according to our needs. For example, when the solution is searched until the third iteration, it can be determined as follows:

$$
x(t) \approx X=x_{0}+x_{1}+x_{2}+x_{3},
$$




$$
\begin{gathered}
y(t) \approx Y=y_{0}+y_{1}+y_{2}+y_{3}, \\
z(t) \approx Z=z_{0}+z_{1}+z_{2}+z_{3} .
\end{gathered}
$$

\section{Results}

We assume to have the following values for the parameters [2]:

$$
\begin{gathered}
c_{1}=\frac{7675406}{7675893}, \quad c_{2}=\frac{487}{7675893}, \quad c_{3}=0.056, \quad \alpha=0.232198, \\
\beta=0.328879, \quad \mu_{\mathrm{h}}=0.0000460, \gamma=0.375 \text { and } \delta_{1}=0.0323 .
\end{gathered}
$$

Therefore, the Adomian initialisation gives

$$
\begin{gathered}
x_{0}=\frac{7675406}{7675893}+\int_{0}^{t} 0.0000460 \mathrm{~d} x=0.999936555+0.0000460 \mathrm{t} \\
y_{0}=\frac{487}{7675893}=0.0000634454 \\
z_{0}=0.056
\end{gathered}
$$

By using Maple software, $x_{1}, y_{1}$ and $z_{1}$ are obtained:

$$
\begin{gathered}
x_{1}=-0.01304826010 t-3.00129024010^{-7} t^{2} \\
y_{1}=0.01298139716 t+2.99071024010^{-7} t^{2} \\
z_{1}=-0.001786340333 t
\end{gathered}
$$

Next, $x_{2}, y_{2}$ and $z_{2}$ are:

$$
\begin{gathered}
x_{2}=0.0002925131156 t^{2}+7.666550135710^{-9} t^{3} \\
y_{2}=-0.002426867464 t^{2}-4.04469591310^{-8} t^{3} \\
z_{2}=0.002326577944 t^{2}+3.52903808310^{-8} t^{3}
\end{gathered}
$$

Finally, $x_{3}, y_{3}$ and $z_{3}$ are:

$$
\begin{gathered}
x_{3}=-0.0001831405726 t^{3}-8.31719574010^{-9} t^{4}-7.53880738010^{-14} t^{5} \\
y_{3}=0.0004491846690 t^{3}+1.16426464610^{-8}+7.53880738010^{-14} t^{5} \\
z_{3}=-0.0003085396521 t^{3}-3.814650368110^{-9} t^{4}
\end{gathered}
$$

The solution to the system of equations (1)-(3) is the sum of the results of all iterations that have been computed. Suppose the sum for $x$ is $X$, the sum for $y$ is $Y$ and the sum for $z$ is $Z$, then the solution is:

$$
\begin{aligned}
X= & 0.999936555-0.01300226010 t+0.0002922129866 t^{2}-0.000183132907 t^{3}- \\
& 8.31719574010^{-9} t^{4}-7.53880738010^{-14} t^{5} \\
Y= & 0.00006344538675+0.01298139716 t-0.002426568393 t^{2}+ \\
& 0.0004491442220 t^{3}+1.16426464610^{-8} t^{4}+7.53880738010^{-14} t^{5} \\
Z= & 0.056-0.001786340333 t+0.002326577944 t^{2}-0.000308504361 t^{3} \\
- & 3.814650368110^{-9} t^{4} .
\end{aligned}
$$


Figure 1 shows the solution of the SIR model of the spread of dengue fever obtained using Adomian decomposition method up to the third iteration. This solution is accurate for small time. For large time, more iterations are needed.

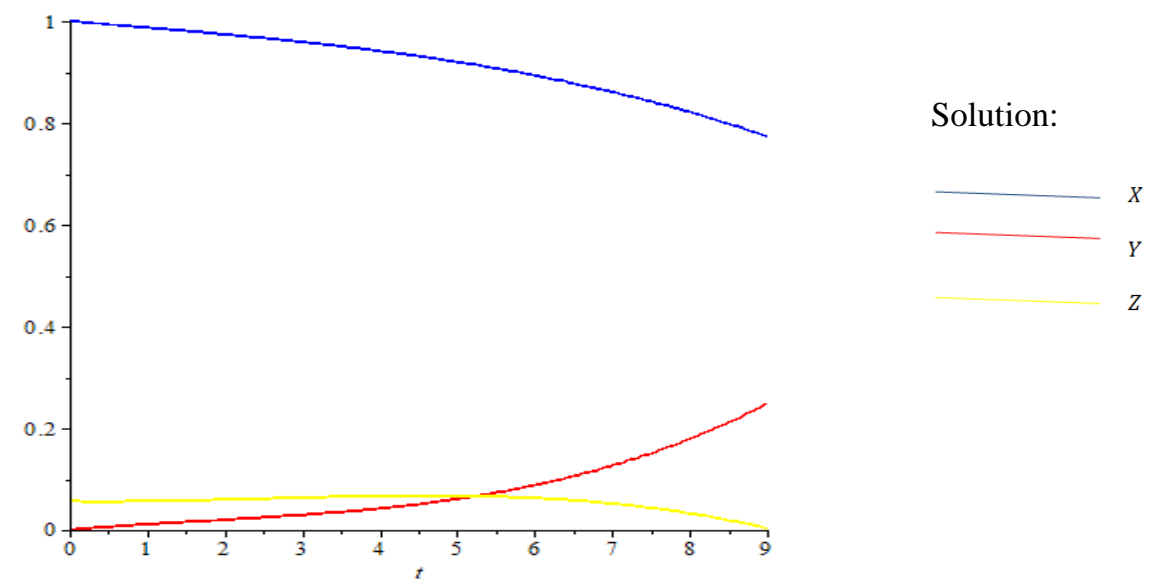

Fig. 1. Solution up to the third iteration of Adomian decomposition method $X, Y$, and $Z$ on $0 \leq t \leq 9$.

\section{Conclusion}

We have solved the SIR model of dengue fever using the Adomian decomposition method. The method produces explicit forms of solutions. Therefore, calculation of the solution is easy to do. This is the advantage of using the Adomian decomposition method in solving mathematical models.

Acknowledgments. The authors thank Universitas Sanata Dharma for the financial support.

\section{References}

[1] Side, S., Noorani, S. M: A SIR model for spread of dengue fever disease (simulation for South Sulawesi, Indonesia and Selangor, Malaysia), World Journal of Modelling and Simulation, Vol. 9, pp. 96-105 (2013)

[2] Rangkuti, Y. M., Side, S., Noorani, M. S. M.: Numerical analytic solution of SIR model of dengue fever disease in South Sulawesi using homotopy perturbation method and variational iteration method. Journal of Mathematical and Fundamental Sciences, Vol. 46, pp. 91-105 (2014)

[3] Putranto, Y. W., Mungkasi, S.: Adomian decomposition method for solving the population dynamics model of two species. Journal of Physics: Conference Series, Vol. 795, Art. 012045 (2017)

[4] Wazwaz, A.M.: Partial Differential Equations and Solitary Waves Theory. Beijing: Higher Education Press and Berlin: Springer (2009) 